\title{
Temperature-triggered formation of a cellulose II nanocrystal network through regioselective derivatization
}

Received 00th January 20xx, Accepted 00th January 20xx DOI: $10.1039 / x 0 x x 00000 x$

\begin{abstract}
Fangbo LIN, ${ }^{a}$ Frédéric PIGNON, ${ }^{b}$ Jean-Luc PUTAUX a and Bruno JEAN *a
The two ends of rodlike cellulose II nanocrystals (CNC-II) were regioselectively functionalized either with gold nanoparticles or thermosensitive polymer chains. In the first case, after the introduction of sulfur atoms at both ends of the rods, a labelling of CNC-II was carried out using a method based on the in situ nucleation and growth of gold nanoparticles (AuNP) from soluble derivatives. Transmission electron microscopy (TEM) images showed that such a method resulted in the grafting of one monodisperse AuNP at each extremity of the CNC-II, i.e. to the formation of hybrid dumbbell-shaped objects. No AuNP was detected on the lateral surfaces of the CNC-II and almost all observed CNC-II exhibited this dual labelling. This result confirmed with a good statistics when compared to previous works the possibility to derivatize only the two ends of the CNC-II, thanks to the antiparallel arrangement of cellulose chains in these nanoparticles. In the second case, the localized grafting of temperature-sensitive macromolecules onto the ends of the CNC-II was performed using an oxidation reaction followed by a peptide coupling. This end-specific grafting of thermosensitive chains onto CNC-II enhanced their colloidal stability when the temperature was below the lower critical solution temperature (LCST) of the polymer. Above the LCST, the TEM images revealed the formation of a network extending to tens of microns resulting from end-to-end associations of the derivatized rods through attractive interactions between collapsed polymer chains. Rheology experiments further evidenced a temperature-induced sol-gel transition from a liquid-like (sol) behavior below the LCST to solid-like (gel) behavior above the LCST, in agreement with a change from purely repulsive interactions to interconnections via the hydrophobic collapsed chains. Importantly, all results concur with a full reversibility of the phenomena upon cooling and reproducibility when samples were subjected to temperature cycles around the LCST. This work reveals that the dual sitespecific derivatization of CNC-II can provide symmetric hybrid particles with innovative assembling and macroscopic properties that cannot be obtained through homogenous chemical modifications.
\end{abstract}

\section{Introduction}

As a renewable resource for biobased materials and fuels, cellulose, a linear polysaccharide composed of $\beta$-Danhydroglucopyranose units, gradually becomes one of the focuses of sustainable development. ${ }^{1}$ One of the specificities of cellulose is that each chain possesses two different termini with a reducing end displaying a hemiacetal cyclic group in equilibrium with an aldehyde moiety and a non-reducing end exhibiting a secondary hydroxyl group. Native cellulose, which is produced via directional biosynthesis by plants, tunicates, algae or bacteria, can be found in the form of slender microfibrils composed of cellulose chains stacked in a parallel fashion. ${ }^{2-4}$ As a result, native cellulose microfibrils inherit the chemical polarity of the constituting chains. Native cellulose, also referred to as cellulose $\mathrm{I}$, is the most studied allomorph but

\footnotetext{
a. Univ. Grenoble Alpes, CNRS, CERMAV, F-38000 Grenoble, France.

Email: bruno.jean@cermav.cnrs.fr

b. Univ. Grenoble Alpes, CNRS, Grenoble INP (Institute of Engineering Univ. Grenoble Alpes), LRP, F-38000 Grenoble, France.

Electronic Supplementary Information (ESI) available: See DOI: 10.1039/x0xx00000x
}

other allomorphs can be prepared using physicochemical treatments. ${ }^{5-9}$ Notably, the cellulose II allomorph can be obtained by a chemical treatment called "mercerization" that is based on the swelling of native cellulose microfibrils in concentrated sodium hydroxide solution and subsequent removal of the swelling agent. ${ }^{10}$ Unlike cellulose I, cellulose II is made of antiparallel chains resulting in a more stable crystal structure. An interdigitation mechanism was proposed to explain the parallel to antiparallel rearrangement of cellulose chains during the cellulose I to II allomorphic transition in fibrous materials: microfibrillar constraints would be released upon swelling in $\mathrm{NaOH}$ and intermingling of chains from neighboring microfibrils of opposite polarity would take place. Upon regeneration and removal of alkali by washing with water, the chains would recrystallize in a more energetically favorable antiparallel arrangement. Therefore, considering the chemical polarity of individual cellulose chains, such an antiparallel arrangement in cellulose II results in the presence of both reducing and non-reducing ends on the two ends of mercerized cellulose fibers.

Cellulose nanocrystals can be isolated from cellulose microfibrils using acid hydrolysis, during which disordered 
regions are preferentially hydrolyzed while crystalline regions remain intact. $^{9}$ Thus, following a sulfuric acid hydrolysis treatment on cellulose II microfibrils, type II rodlike cellulose nanocrystals can also be produced. They will be referred to as CNC-II in the following, while CNC-I will be used for nanocrystals derived from native cellulose. Transmission electron microscopy (TEM) was used by different groups to show that both CNC-I and CNC-II extracted from ramie, cotton or eucalyptus exhibit a rodlike morphology and are composed of a few laterally-bound elementary crystallites. ${ }^{11-13}$ However, as also confirmed by other authors using scanning electron microscopy or atomic force microscopy, ${ }^{14-15}$ CNC-II prepared from the acid hydrolysis of mercerized cellulose are systematically shorter than their cellulose I counterpart, while their width and height are rather identical. As a consequence, CNC-II have a lower aspect ratio than CNC-I. Alternatively, Hirota et al. prepared cellulose II nanocrystals from mercerized wood or cotton cellulose by TEMPO-mediated oxidation at $\mathrm{pH}$ 4.8 or 9 , in some cases after acid hydrolysis. They obtained stable suspensions of individualized cellulose II nanocrystals with a high carboxylate content and a width comparable to native cellulose nanocrystals. ${ }^{16-17}$ Gong et al. showed that TEMPO oxidation of CNC-II prepared from the acid hydrolysis of mercerized microcrystalline cellulose increases the colloidal stability of the particles, while keeping their morphology unchanged. ${ }^{18}$ Other pathways for the preparation of cellulose II nanocrystals were explored by Sèbe et al. ${ }^{19}$ and Jin et al. ${ }^{20}$ In the first case, cellulose II nanocrystals were prepared from cellulose I substrates without any mercerization step using dropwise addition of given amounts of concentrated sulfuric acid. The obtained particles exhibited a ribbon-like morphology with a rounded shape at the tip and a reduced length when compared to their cellulose I homolog. However, in contrast to the CNC-I described above, the molecular direction of the cellulose chains in these particles is not aligned parallel to the long axis of the crystals, but perpendicular to it. ${ }^{12,21-22}$ In the second case, the authors investigated the allomorphic and morphological changes upon mercerization of cellulose I nanocrystals. The results indicated a gradual allomorphic transition from cellulose I to cellulose II in a certain range of alkali concentrations and TEM observation revealed that after mercerization the crystals tend to associate and form some interconnected granular structures. Alternatively, $\mathrm{Yu}$ et al. produced cellulose II nanospheres from the hydrolysis of lyocell fibers with mixed acids (90\% formic acid and $10 \%$ hydrochloric acid). ${ }^{23}$ These works show that non-conventional preparation protocols result in various shapes and surface characteristics of the CNC-II.

Beyond the investigation of the preparation and morphological features of CNC-II, a few studies were devoted to the study of their colloidal properties. Li et al. showed that CNC-II exhibited a lower ability to stabilize oil in water emulsions than CNC-I.24 Interestingly, Delepierre and coworkers recently showed that
CNC-II suspensions could very slowly self-organize into longpitch ( $54 \mu \mathrm{m}$ for a $8 \mathrm{wt} \%$ suspension) cholesteric phases, while CNC-I suspensions obtained from the same source formed much lower pitch (8.7 $\mu \mathrm{m}$ for a $8 \mathrm{wt} \%$ suspension) chiral nematic phases after shorter equilibration time. ${ }^{14}$ Such a result was attributed to the lower aspect ratio and higher diffusion coefficient of CNC-II, while the difference in twist frequency and electric dipole moment could also play a role. The potential of CNC-II to be used as templates for the fabrication of porous $\mathrm{SiO}_{2}$ particles with smaller and narrower pore width distribution when compared to materials synthesized using CNC-I was demonstrated by Kuang et al. ${ }^{25}$ Globally, these recent results emphasize that the use of CNC-II is a way to tune the properties of CNC-based materials, which could help targeting specific applications for which CNCs-I would not be suitable.

In our previous work, based on the chemical polarity of cellulose $\mathrm{I}$, a regioselective grafting of thermosensitive polymer chains at the reducing end of CNC-I could be successfully achieved from consecutive oxidation and peptide coupling reactions. The obtained derivatized objects were able to reversibly form starshaped complexes via a simple control of temperature. ${ }^{26}$ Challenges and new properties provided by the chemical modification of the reducing end of CNC-I have recently been exposed in two reviews. ${ }^{27-28}$ In contrast with CNC-I, the presence of aldehyde groups on both ends of CNC-II should allow functionalizing both extremities. However, to date, such a regioselective dual derivatization has received very little attention. To our knowledge, only Kim et al. have reported the successful labeling of both ends of CNC-Il from mercerized ramie cellulose with gold nanoparticles (AuNPs), using a biotin/streptavidin strategy. However, the TEM micrographs shown in this work revealed that only a minor fraction of CNC-II were labeled at both ends, while other particles were labeled at only one end or not labeled at all.

In the present paper, we first aimed at efficiently labelling the two ends of CNC-II with AuNPs. To reach that goal, we have transposed to the case of CNC-II a recently developed strategy that allowed us to label the reducing end of CNC-I with AuNPs with a very high labeling yield of about $80 \%$. Such a method is based on the in situ nucleation and growth of AuNPs in presence of CNCs bearing sulfur atoms at the cellulose chain reducing ends. The second objective was to graft thermosensitive polymer chains at both ends on CNC-II. The morphological features and assembling properties of these innovative nanoparticles were investigated as a function of temperature (below and above the lower critical solution temperature, LCST, of the thermosensitive polymer) using dynamic light scattering (DLS) and TEM. In addition, the rheological properties of these regioselectively derivatized cellulose II nanocrystals were assessed through rheometric measurements carried out in steady states or oscillatory tests to access to viscosity and viscoelasticity moduli, respectively. To our knowledge, this work 
is the first report on the regioselective derivatization of CNC-I with stimuli-sensitive macromolecules.

\section{Results and discussion}

\section{Characterization of CNC-II particles}

The physicochemical properties and morphology of the particles prepared from cotton linters following the mercerization and sulfuric acid hydrolysis protocol were first investigated by various experimental techniques. The wide-angle powder X-ray diffraction pattern of CNC-II shown in Supplementary Information Figure S1 contains three main diffraction rings at diffraction angles $2 \vartheta=12.5$, 20.5 and $22.5^{\circ}$ that correspond to the (110), (110) and (020) planes of the cellulose II crystal. This demonstrates that the mercerization resulted in the allomorphic conversion from cellulose I to cellulose II. ${ }^{29-30}$ The FTIR spectrum of CNC-II shows the presence of two distinctive peaks at about 3490 and $3450 \mathrm{~cm}^{-1}$, which correspond to intramolecular hydrogen bonding in cellulose II (Supplementary Information Figure S2). Another evidence for the conversion of cellulose I into cellulose II is provided by the significant decrease of the peak intensity at $1430 \mathrm{~cm}^{-1}$ due to the conformation change of hydroxymethyl group from $\mathrm{tg}$ to $\mathrm{gt}$ form. The stretching vibration of $\mathrm{C}-\mathrm{O}$ at $\mathrm{C} 6$ peak was shifted to $1030 \mathrm{~cm}^{-1}$ as the torsional angles of $\beta$ $(1,4)$-D-glycosidic linkages changed. Consequently, the C-O-C vibration of this linkage was switched to $895 \mathrm{~cm}^{-1}$. These results agree with literature reports. ${ }^{31-34}$ The ${ }^{13} \mathrm{C} C P / M A S$ solid-state NMR spectrum of CNC-II shows an increase in the intensity of the peak near 107.3 ppm, which is attributed to carbon C1 of the glucosyl units in the crystalline part of cellulose II (Supplementary Information Figure S3). Moreover, the disappearance of the signal at $65 \mathrm{ppm}$, associated with the crystalline regions of cellulose I, also proved the transition from cellulose I to cellulose II. The peak at $76 \mathrm{ppm}$, attributed to $\mathrm{C2}, \mathrm{C} 3$ and $\mathrm{C} 5$ carbons, reveals variations in hydrogen bonding patterns resulting from a new molecular conformation of the chains. These results are also in good agreement with the literature reports. ${ }^{34-35}$

The morphology and dimensions of the CNC-II were characterized by TEM imaging (Figure 1a) and SANS experiments (Figure 1b). As shown in Figure 1a, CNC-II are rodlike particles with a length ranging between 50 and $150 \mathrm{~nm}$ and an average width of $16 \mathrm{~nm}$, even if some wider particles can be observed. They are therefore shorter than cotton CNC-I produced from the same cellulose source, while having a more or less comparable width. ${ }^{36-37}$ CNC-II extracted from cotton therefore exhibit an aspect ratio about 6 , which is lower than that of their cellulose I homolog ( 10). Like CNC-I, they are composed of a few laterally-associated elementary crystallites. These morphological features are in agreement with those reported for CNC-II obtained from ramie, cotton or eucalyptus. ${ }^{11-15}$ Figure $1 \mathrm{~b}$ shows the SANS spectrum of a $1 \mathrm{wt} \% \mathrm{CNC}$-II suspension in $\mathrm{D}_{2} \mathrm{O}$. A very clear $Q^{-4}$ decay can be observed in the high- $Q$ region (from $Q$ $\left.0.05 \AA^{-1}\right)$. Such a Porod law behavior readily shows that the CNC-II- water interface is sharp and smooth and arises from the crystalline and smooth character of all the facets of the particles. In the low $Q$ region, a plateau is observed and a shoulder can be detected at $Q^{*} \sim$ $6.510^{-3} \AA^{-1}$. The latter feature can be attributed to the electrostatic repulsion between particles giving rise to some contribution of the structure factor to the scattered intensity. The origin of this electrostatic interaction is the presence of sulfate half-esters resulting from the sulfuric acid hydrolysis (see below). The position of this peak can be used to calculate the average interparticle distance, $d$, from $d=2 \pi / Q^{*}$. A $d$ value of $97 \mathrm{~nm}$ can thus be calculated. As shown in various studies, SANS data of dilute CNC suspensions have been well-described using a model based on the form factor of a parallelepiped with a rectangular cross-section. ${ }^{36,38-}$ 40 A similar model was used here since literature data and TEM images show that CNC-I and CNC-II exhibit the same morphology but with different dimensions. As shown in Figure $1 b$, a good agreement between the fit and the experimental spectrum was obtained for $Q$ $>110^{-2} \AA^{-1}$. Nevertheless, as the fitting process did not take into account the influence of the structure factor whose contribution is observed at low $Q$-values, the model only fairly fits the data in the low $Q$-range. The best fit was obtained with values of 74.0, 21.5 and $3.6 \mathrm{~nm}$ for the length, width and thickness, respectively, using a polydispersity of 0.05 on the width and 0.32 on the thickness. In addition to the overall good fitting quality, these average length and width values are in good agreement with our TEM data and more generally with dimensions reported in the literature. The thickness of $3.6 \mathrm{~nm}$ is smaller than the one measured by atomic force microscopy images by Delepierre et al. from height profiles $(5 \pm 1$ $n m) .{ }^{14}$ Knowing the dimensions of the rods and the volume fraction, and assuming a close packing, an average distance of $109 \mathrm{~nm}$ between the center of mass of the particles can be calculated. This value is close to that independently obtained from the intensity maximum at $Q^{*}$ and therefore confirms the validity of the dimensions extracted from the fit.

A sulfur content (S\%) of $0.67 \%$ was measured by conductometric titration which is very close to the value reported for cotton CNC-I (0.69\%). ${ }^{36-37}$ This sulfur content provided by sulfate groups on the surface of the nanorods corresponds to a surface charge density of $0.3 \mathrm{e}^{-} \mathrm{nm}^{-2}$, which is a little lower than that of CNC-I. ${ }^{26}$ The zeta potential was $-32.1 \pm 4 \mathrm{mV}$. Both the sulfur content and zeta potential values are comparable with those reported for CNC-II extracted from eucalyptus or cotton and confirm that CNC-I and CNC-II display similar surface characteristics. ${ }^{12}$

\section{Identification of the chemical symmetry of CNC-II}

The labeling of the two ends of CNC-II with AuNPs was previously addressed by Kim et al. and used to show that in CNCII from mercerized ramie cellulose, the chains were packed into an antiparallel fashion. ${ }^{11}$ However, as noted by these authors, only a small percentage of about $15 \%$ of these CNC-II were labeled at both ends. In this work, prior to the regioselective grafting of thermosensitive polymer chains, we aimed at 
verifying the availability and location of the reactive aldehyde sites using the same type of labeling, but relying on an alternative approach we recently developed with CNC-I. Indeed, using a strategy that consisted in synthesizing AuNPs in situ

from soluble gold derivatives in the presence of CNCs functionalized at their reducing end with thiosemicarbazide molecules, we could achieve unprecedent high labeling yields with a vast majority of CNC-I bearing one or several AuNPs at their reducing end. The same two-steps method described in the experimental section was thus transposed to CNC-II.
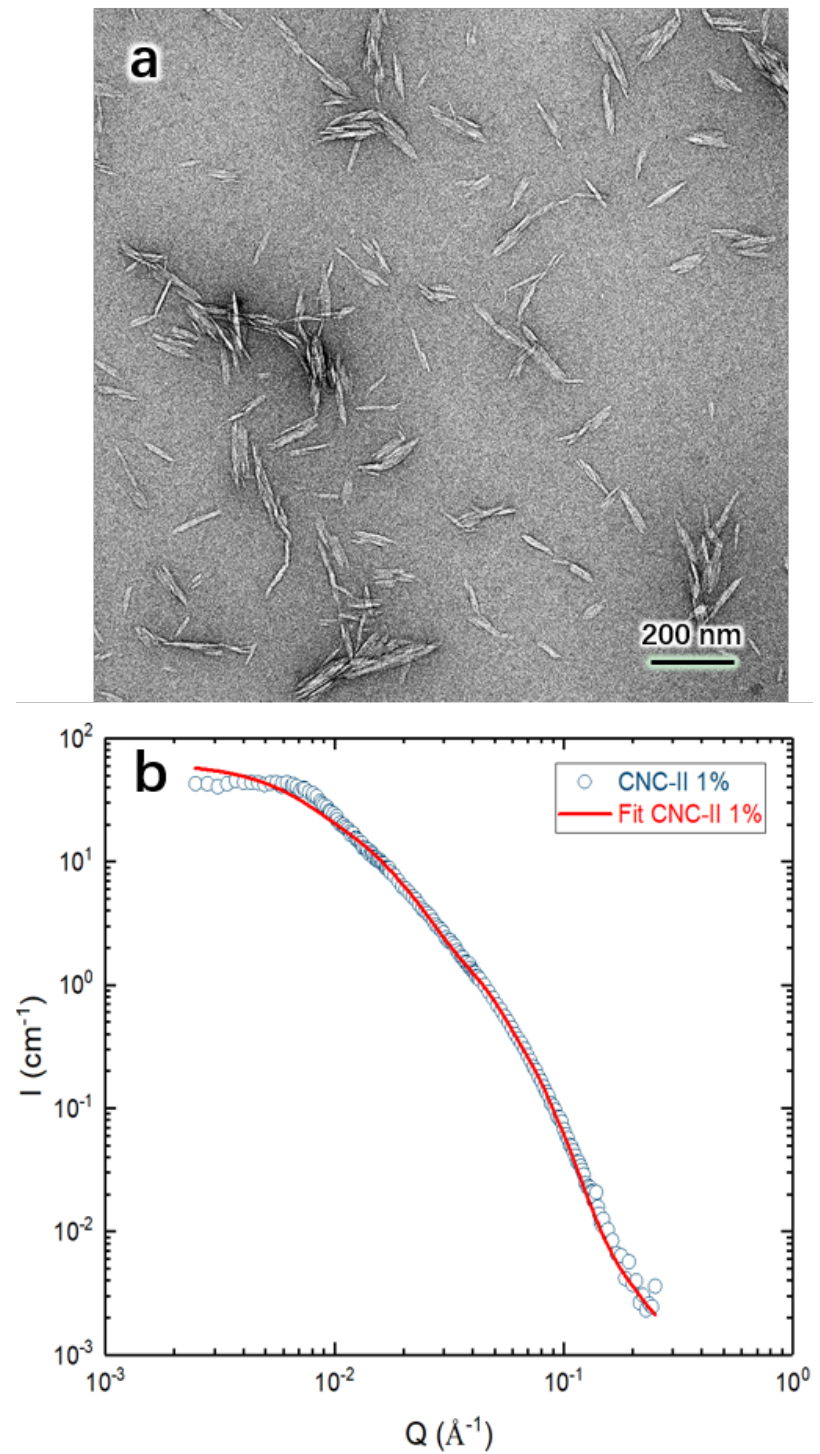

Figure 1. a) TEM micrograph of negatively stained CNC-II prepared from an aqueous suspension; b) SANS spectrum of a 1 wt \% CNC-Il suspension in $\mathrm{D}_{2} \mathrm{O}$ and fit using the form factor of a parallelepiped with dimensions $74 \times 21.5 \times 3.6 \mathrm{~nm}^{3}$. A Gaussian polydispersity of 0.05 and 0.32 were applied on the width and thickness, respectively.

First, the CNC-II were treated with thiosemicarbazide in the presence of acetic acid to introduce sulfur atoms at the cellulose chains reducing ends through a reaction with the aldehyde groups. This first reaction was quantitatively characterized by measuring the aldehyde content before and after this prefunctionalization reaction using Fehling's test. Precisely, the aldehyde content decreased from $22.7 \pm 0.5 \mu \mathrm{mol} \mathrm{CHO} \mathrm{g}^{-1}$ for as-prepared CNC-II to $8.1 \pm 0.5 \mu \mathrm{mol} \mathrm{g}^{-1}$ after the reaction with thiosemicarbazide, revealing a conversion rate of about $64 \%$. The nucleation and growth of AuNPs from soluble derivatives was then carried out in presence of these sulfur-containing CNC-II and the resulting sample was characterized by TEM and dynamic light scattering (DLS). As shown in Figure 2, TEM images show the prominent formation of "dumbbells" consisting of one CNC-II with one AuNP grafted on both ends. In contrast with Kim and coworkers' conclusion, our method resulted in a very high labeling efficiency since almost all CNC-II particles exhibited a single AuNP on both ends.
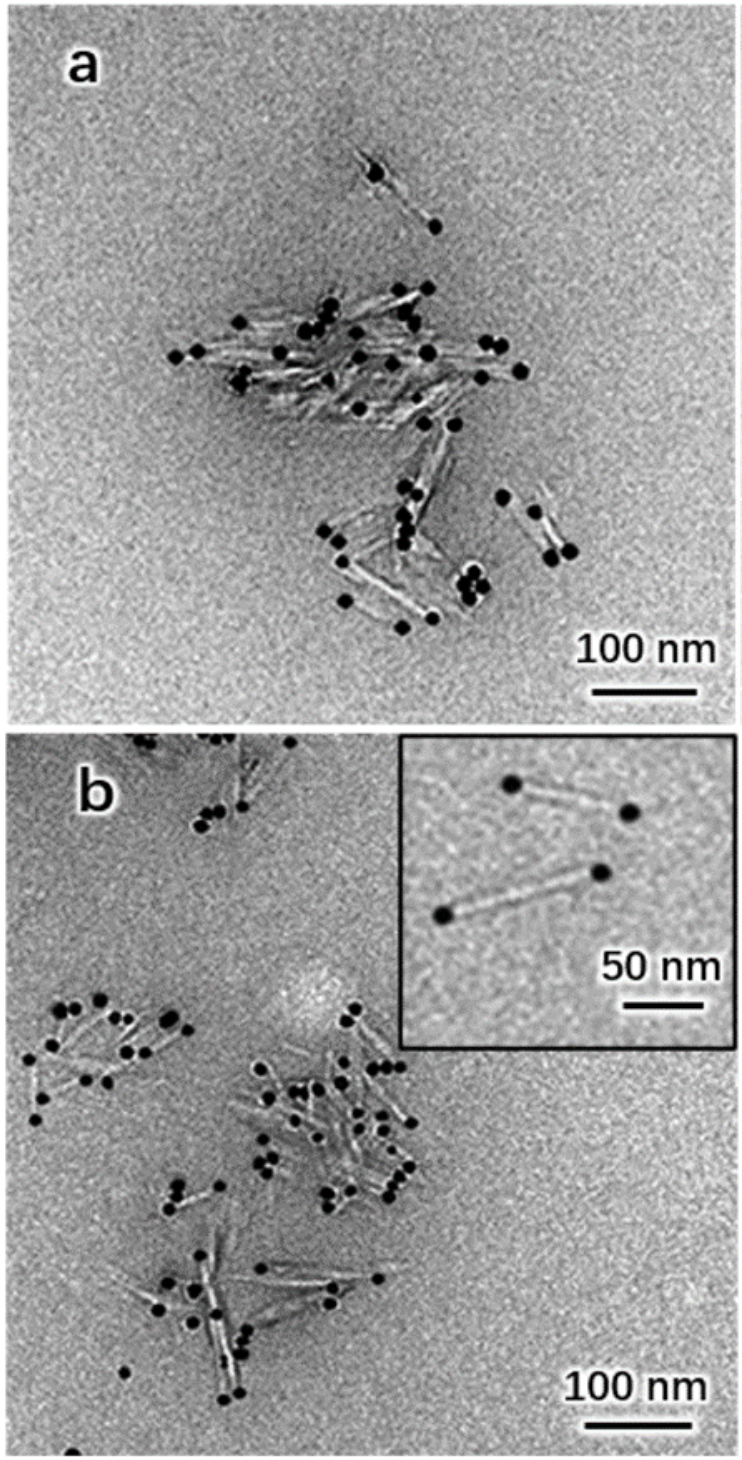

Figure 2. TEM images of negatively stained AuNP-e-CNC-II prepared by in situ growth from aqueous suspensions at room temperature. The insert in $\mathrm{b}$ shows isolated CNC-II-AuNP dumbbells. 
Such a result gives a statistically relevant visual evidence for the antiparallel character of cellulose chains in CNC-II. It additionally proves that the aldehydes are only located at the rod ends, since no AuNP was observed at the lateral side of the CNC-II, which suggests that a true regioselective modification of the CNC-II targeting only their two ends is possible. It also has to be noted that the attached AuNPs exhibit a very narrow size dispersity. DLS experiment were carried out on sulfur-functionalized CNCII and AuNP-labeled CNC-II suspensions and compared with data from a pure (CNC-free) AuNP suspension synthesized in the same conditions as described in the experimental section. Results are shown in Figure 3. The average hydrodynamic diameter of sulfur-functionalized CNC-II is $122 \mathrm{~nm}$ while that of independently-prepared AuNPs is $20 \mathrm{~nm}$. After the in situ growth reaction, a bimodal distribution is observed with a very low-intensity peak at $20 \mathrm{~nm}$ and a high-intensity one at $154 \mathrm{~nm}$. The first signal can tentatively be attributed to the presence of a limited number of individual (not attached to CNCs) AuNPs, while the second one reasonably stands for AuNP-labeled CNCII, exhibiting a significantly higher diameter than the asprepared CNC-II (32 $\mathrm{nm}$ increase). Such features are fully consistent with TEM observations.

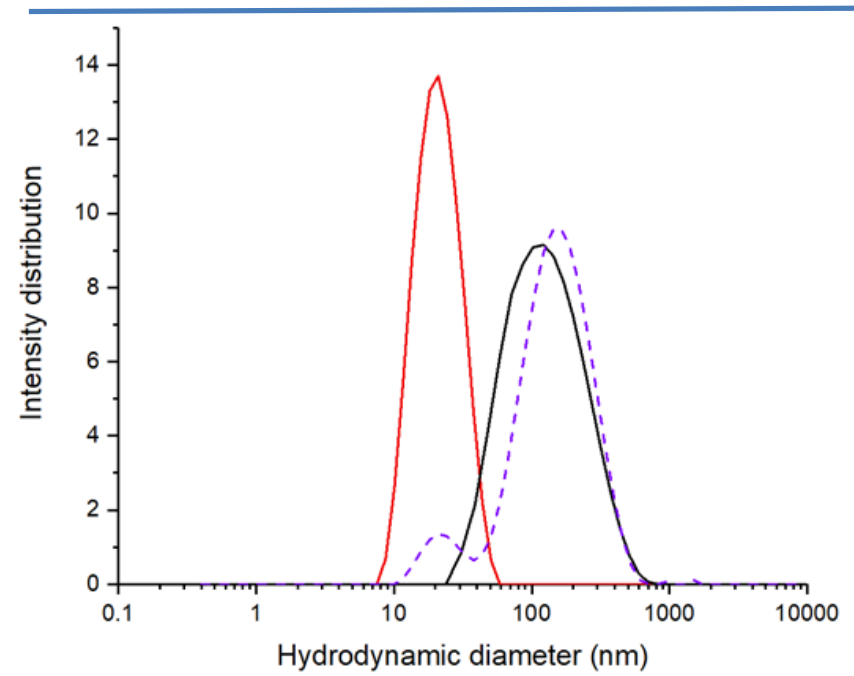

Figure 3. Intensity size distribution of a $0.5 \mathrm{wt} \%$ gold nanoparticle suspension (red solid line), 0.1 wt \% CNC-II-e-S suspension (black solid line) and 0.1 wt \% AuNP-e-CNC-II suspension (purple dashed line) prepared by in situ growth and measured by DLS at $20^{\circ} \mathrm{C}$.

\section{Two-step regioselective functionalization of CNC-II with thermosensitive polymer chains}

In order to graft thermosensitive polymers onto both ends of CNC-II particles, a two-step protocol described in Scheme 1 was followed. First, the aldehyde groups present at both extremities were converted into carboxylates by a chlorite oxidation method and the resulting particles will be referred to as Ox-eCNC-II. The aldehyde contents before and after oxidation reaction could be measured by the Fehling test. The initial and post oxidation values were $22.7 \pm 0.5 \mu \mathrm{mol} \mathrm{CHO} \mathrm{g}^{-1}$ and $5.6 \pm 0.4$ $\mu \mathrm{mol} \mathrm{CHO} \mathrm{g}{ }^{-1}$, respectively, proving the success of the oxidation reaction with a yield of about $75 \%$. The polyetheramine T5000 was then grafted onto Ox-e-CNC-II by a peptide coupling reaction. In the presence of catalysts, the amino groups of the polymer chains and the carboxylated groups on the rod ends condensed to form an amide bond. Such a regioselective grafting only concerns a very small number of anhydroglucose (AGU) units (only those at the ends of the rods, representing only a few percent of the total AGU units), which made attempts to quantity the functionalization yield by FTIR or CP/MAS NMR unsuccessful.

\section{Temperature-triggered assembling properties of polymer- grafted CNC-II}

In order to investigate the effect of the regioselective grafting of polyetheramine onto the ends of CNC-II, DLS was used to measure the hydrodynamic diameter $\left(D_{h}\right)$ as a function of temperature for unmodified CNC-II, Ox-e-CNC-II and polymergrafted CNC-II (T5000-e-CNC-II). As shown in Figure 4a, CNC-II presents a constant $D_{h}$ at about $120 \mathrm{~nm}$, which is very close to the hydrodynamic diameter of end-oxidized CNC-II, showing, as expected, no significant influence of chlorite oxidation. Similarly, within the temperature range of $4-24{ }^{\circ} \mathrm{C}, D_{h}$ of asprepared CNC-II and Ox-e-CNC-II remained constant showing no temperature effect on these two nanoparticles. Then, for T5000-e-CNC-II, when the temperature was lower than the LCST of the polyetheramine, a constant value of about $150 \mathrm{~nm}$ was measured. This value, that is significantly higher than the one for CNC-II and Ox-e-CNC-II, is attributed to the presence of polyetheramine chains on the ends of the rods, which slows down their dynamics, and is therefore an indirect evidence of the success of polymer grafting. It must be noted that this increase of hydrodynamic diameter after polyetheramine grafting could also come from the formation of duplex or triplex particle associations because of the use of the polyetheramine T5000 that is a triamine, which theoretically allows for connecting two or three CNC-II. However, such a reaction is unlikely due to steric hindrance and no such duplex or triplex structures were observed by TEM (vide supra).

In contrast with the constant value measured below the LCST, a significant increase in hydrodynamic diameter was observed when the sample was heated above this critical temperature. Indeed, $D_{h}$ increased from 150 to about $1200 \mathrm{~nm}$ corresponding to the formation of aggregates. This value is much higher than the one measured in the case of temperature-induced formation of star-shaped complexes formed by CNC-I whose reducing end was derivatized with thermosensitive chains. ${ }^{26}$ When the temperature decreased from above the LCT to $4^{\circ} \mathrm{C}$, Dh spectacularly decreased until it reached back the initial diameter of about $150 \mathrm{~nm}$, indicating the complete dissociation of the aggregates. This result reveals a fully thermoreversible behavior characterized by a hysteresis phenomenon related to a faster association than dissociation. In fact, when temperature cycles between 4 and $24{ }^{\circ} \mathrm{C}$ were repeated, this thermoreversible behavior was totally reproducible (Figure $4 b$ ). Even though the suspension experienced numerous temperature variations conditions, the reversible temperatureinduced aggregation was preserved. 


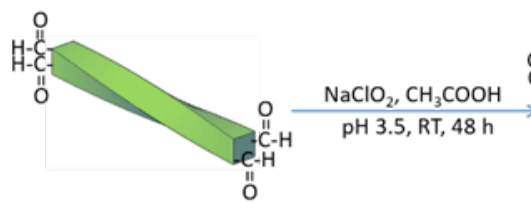

CNC-II

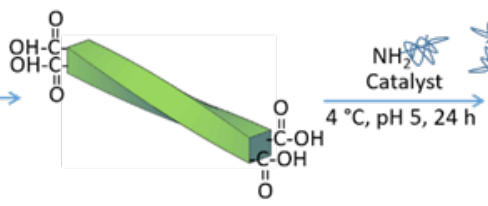

Ox-e-CNC-II

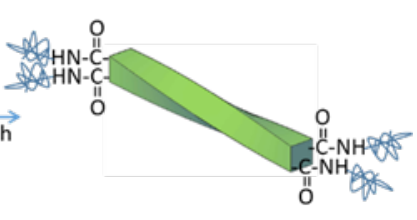

Polyetheramine-e-CNC-II

Scheme 1. Two-step functionalization of CNC-II with Jeffamine ${ }^{\circledR}$ polyetheramine chains in aqueous medium. After a carboxylation of the reducing ends, the grafting of the polyetheramine chains was achieved via a peptide coupling reaction.
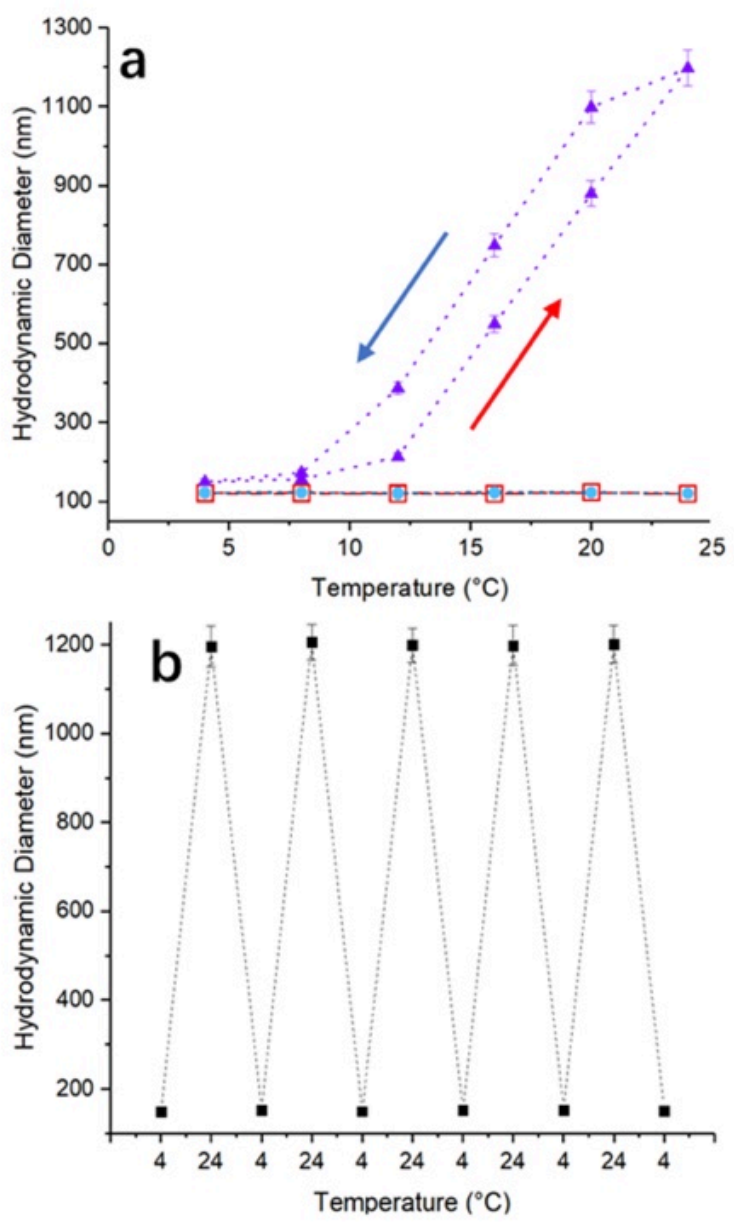

Figure 4. a) Hydrodynamic diameter as a function of a temperature cycle between 4 and $24{ }^{\circ} \mathrm{C}$ for CNC-II (red open squares), Ox-e-CNC-II (cyan dots) and T5000-e-CNC-II (purple triangles) in $0.1 \mathrm{wt} \%$ aqueous suspensions. Heating (red arrow) and cooling (blue arrow) are indicated by arrows. b) Hydrodynamic diameter variations for T5000-e-CNC-II in $0.1 \mathrm{wt}$ $\%$ aqueous suspensions during multiple cycles of temperature increase and decrease between 4 and $24^{\circ} \mathrm{C}$.

To further characterize the temperature-induced aggregation revealed by DLS, samples prepared at 4 and $24{ }^{\circ} \mathrm{C}$ were observed by TEM. Figure 5 shows images of Ox-e-CNC-II after oxidation at 4 and $24^{\circ} \mathrm{C}$. Ox-e-CNC-II nanoparticles shown in

Figure 5a are almost identical to their parent CNC-II, indicating that the chlorite oxidation did not have any effect on the morphology of the particles. When the temperature was increased to $24{ }^{\circ} \mathrm{C}$ (Figure $5 \mathrm{~b}$ ), similar observations were made, showing that a temperature increase had no effect on Ox-eCNC-II, which confirmed the DLS measurements.
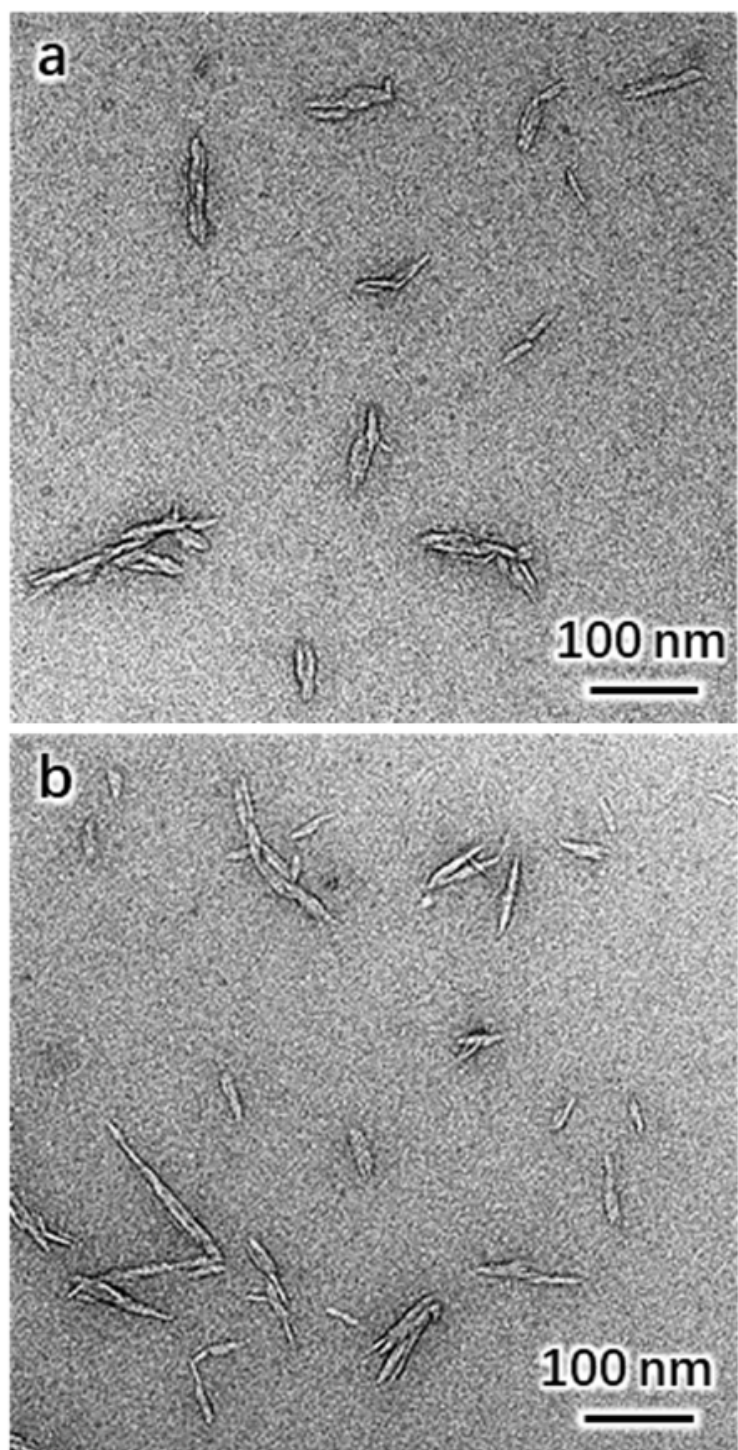

Figure 5. TEM micrographs of negatively stained Ox-e-CNC-II at $4{ }^{\circ} \mathrm{C}(\mathrm{a})$ and $24^{\circ} \mathrm{C}(\mathrm{b})$ prepared from an aqueous suspension.

The TEM micrograph of the T5000-e-CNC-II sample prepared from a suspension kept at $4{ }^{\circ} \mathrm{C}$ is shown in Supplementary Information Figure S4a. These polymer-grafted CNC-II are welldispersed and exhibit the same morphological features as their parent particles (Figure 5). The T5000 chains are not visible, 
which is expected due to their very small size, even though their presence contributes to the hydrodynamic diameter. Additionally, despite the use of triamine, no complexes associating two or three around a unique tri-aminated macromolecule were observed.

As shown in Figure 6, peculiar assemblies of T5000-e-CNC-II were formed from the same sample prepared at $24^{\circ} \mathrm{C}$. Upon temperature increase above the LCST, the derivatized CNC-II indeed self-assembled into a network where the particles are hooked by their ends. Using a lower magnification (Figure 6c), the observed network appears to extend over several micrometers. This spectacular phenomenon is due to the presence of thermosensitive polymers on the two ends of the CNC-II rods.
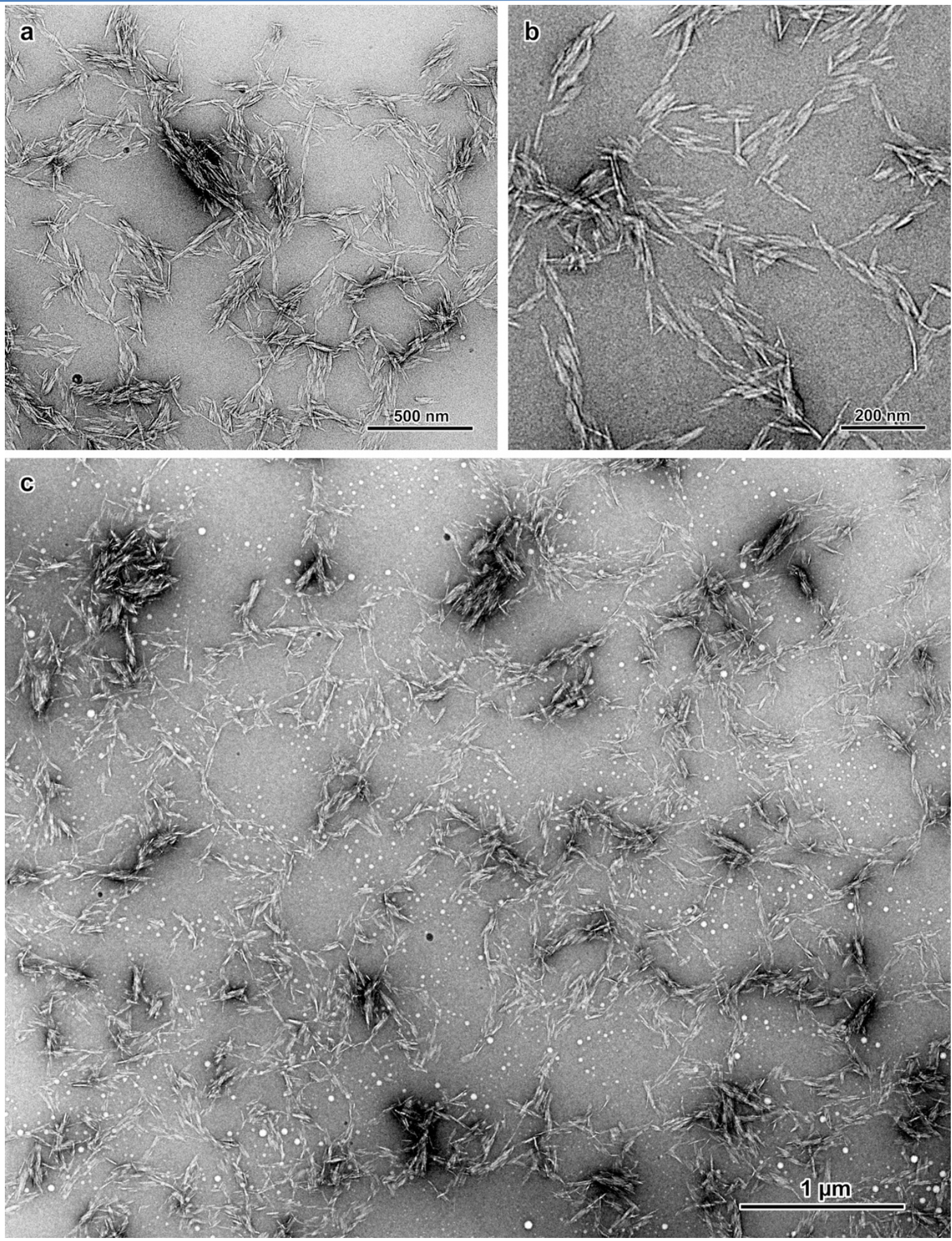

Figure 6. TEM images of negatively stained T5000-e-CNC-II at $24^{\circ} \mathrm{C}$ prepared from aqueous suspensions. 
When the temperature increases above the LCST of the thermosensitive polymer, the solvent conditions become poor and polymer chains collapse. This switch in conformation is accompanied by a change from entropic repulsions below the LCST to attractive interactions mediated by hydrophobic polymer chains above the LCST. Sulfate charges on the side of CNC-II are not affected by variations in temperature in that range and lateral repulsive interactions are preserved. In addition to the presence of interconnected loops made of endto-end linked derivatized CNC-II, some lateral associations are also observed. The latter assembling could result from connections involving both ends of the particles that overcome the electrostatic lateral repulsions. This organization might also arise from artefacts during TEM sample drying. Accordingly, above the LCST, T5000-e-CNC-II assemble through their ends and occasionally via their lateral surface to form the observed network structure, as shown in Scheme 2.

Figure $6 \mathrm{c}$ shows that the network connects a majority of T5000e-CNC-II and that only a very small number of CNCs stay individualized. Such a feature is an indirect proof of a successful derivatization of both ends of the particles with a very high yield. In our previous work, native CNCs were functionalized with thermosensitive polymers at their reducing end, allowing the formation of star-shaped complexes above the LCST. Here in contrast the regioselective dual derivatization increases the association probability, resulting in the temperature-triggered assembling of a very large number of objects. It must be noted that these networks could spontaneously dissociate when the temperature was decreased below the LCST, as shown by TEM images of a sample first heated to $24{ }^{\circ} \mathrm{C}$ and then cooled down to $4{ }^{\circ} \mathrm{C}$ prior to TEM observation (Supplementary Information Figure S4b), in full agreement with the reversibility shown by DLS measurements during temperature cycles.

\section{Rheological behavior of regioselectively-derivatized CNC-II particles}

Derivatized CNC-II and their assembly into networks were well characterized by DLS and TEM in dilute conditions. However, the samples for TEM were prepared by drying and staining the specimens which may induce artefacts. To get more volume information on these systems, a series of rheological experiments including the investigation of the viscosity and viscoelasticity of the suspensions were carried out.

First, flow curves of T5000-e-CNC-II suspensions at concentrations from 1 to 5 wt $\%$ were recorded at $8{ }^{\circ} \mathrm{C}$, well below the LCST of the polyetheramines (Figure 7). Macroscopically, at such a temperature, all samples were fluidlike (sol) and no gel was formed. Clearly, the viscosity of all five samples decreased when the shear rate increased indicating a shear thinning behavior. When compared with CNC-II samples (Supplementary Information Figure S5), 1 and 2 wt \% T5000-eCNC-II suspensions behave similarly and no significant difference was observed.
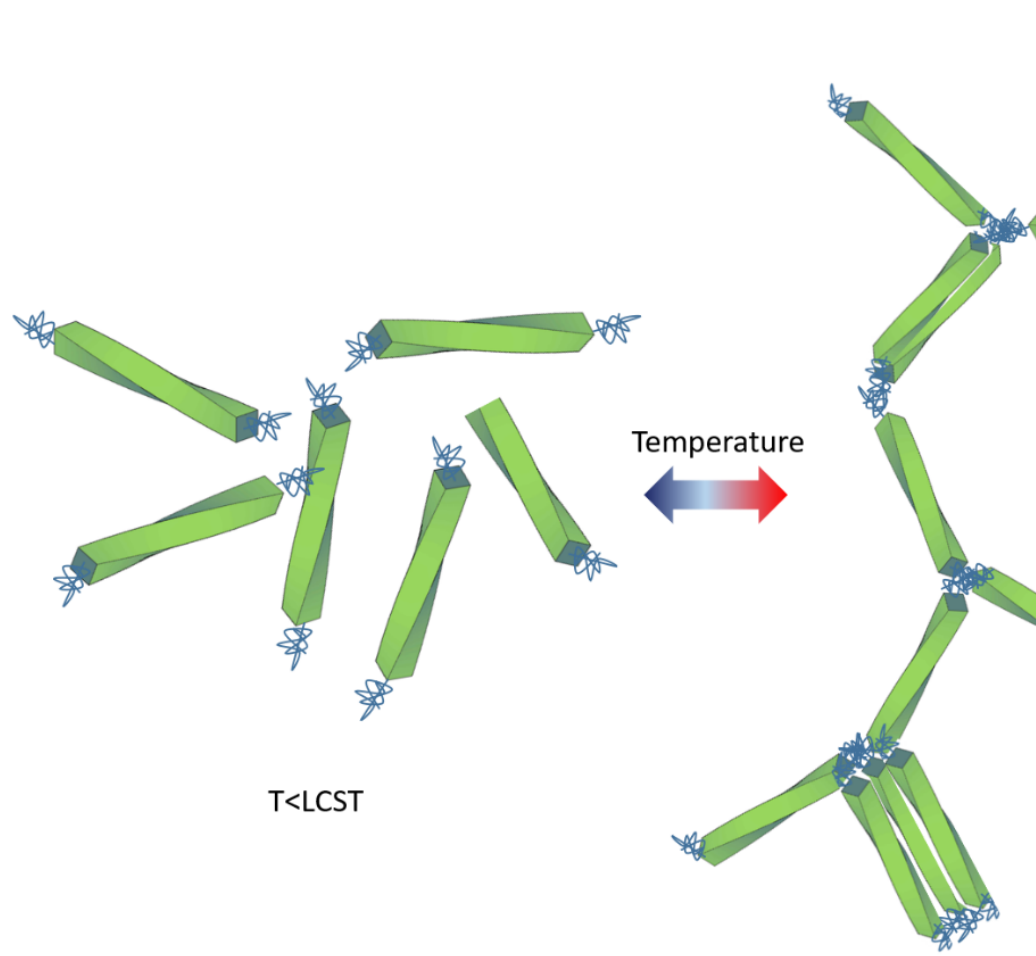

T $>$ LCST

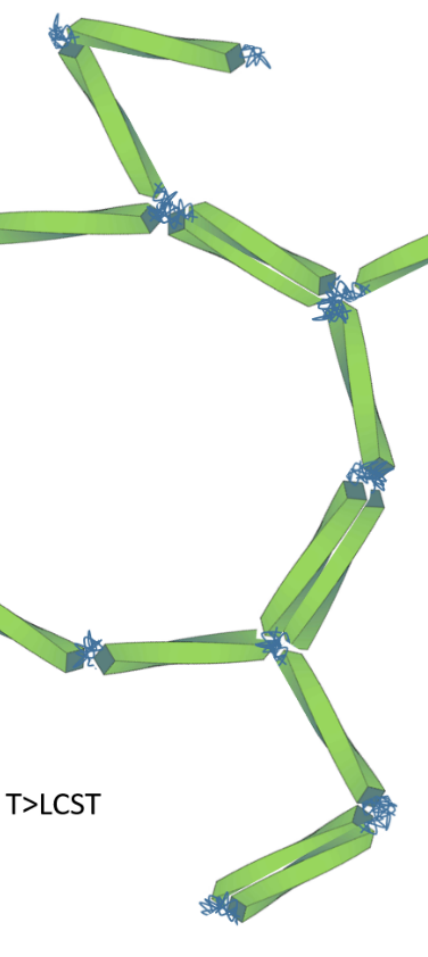

Scheme 2. Thermoreversible association of polyetheramine-e-CNC-II into a network. 
However, the viscosity of 3, 4 and 5 wt \% T5000-e-CNC-II samples is lower than that of unmodified CNC-II samples at the same concentration, in agreement with macroscopic visual observations Supplementary Information Figure S6) showing that as-prepared CNC-II suspensions at 3, 4 and $5 \mathrm{wt} \%$ are gellike. This result can be ascribed to the presence of polyetheramine chains onto the ends of the rods in the case of T5000-e-CNC-II samples that generate entropic repulsive forces when the polymer is in good solvent conditions. Such interactions, which are not present in the case of as-prepared CNC-II, contribute to a better individualization of the nanoparticles and prevent connections to take place, favoring a liquid-like behavior.

Temperature effects on the viscosity and viscoelasticity of a 5 wt \% T5000-e-CNC-II suspension are shown in Figure 8 in the 8$24{ }^{\circ} \mathrm{C}$ range. Viscosity measurements were performed at a shear rate of $30 \mathrm{~s}^{-1}$. This shear rate value of $30 \mathrm{~s}^{-1}$ was selected to reach steady state in a short time at each temperature change. The goal was to get a viscosity value at each temperature with a time dependent effect as short as possible compared to the temperature dependence effect. It was first checked that the viscosity behavior of the underivatized CNC-Il suspensions (in the absence of thermosensitive polymer) measured in the same conditions is temperature independent, as shown in Supplementary Information Figure S7a. For the polyetheramine end-grafted samples, below the LCST, at $8{ }^{\circ} \mathrm{C}$, the viscosity was about 0.005 Pa.s, which corresponds to the value in the flow curve in Figure 7. At $12{ }^{\circ} \mathrm{C}$, i.e. at the LCST of the T5000 polyetheramine, a moderate increase in viscosity was detected, indicating the start of conformation changes of the polymer chains. When $\mathrm{T}>12^{\circ} \mathrm{C}$, the viscosity strongly increased to about 180 Pa.s and tended to reach a plateau, suggesting the formation of connections between the nanocrystals, which is in good agreement with the network structure revealed by TEM images. A hysteresis effect was observed when the sample was

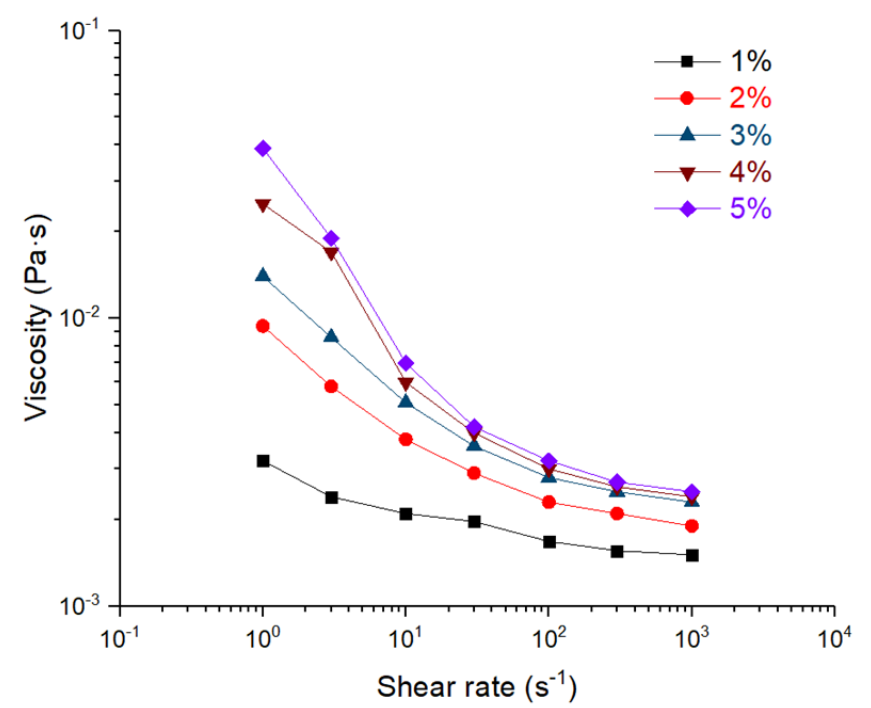

Figure 7. Flow curves of T5000-e-CNC-II samples at concentrations of $1,2,3,4$ and 5 wt $\%$ at $8{ }^{\circ} \mathrm{C}(<$ LCST $)$ in a shear rate range from 1 to $1000 \mathrm{~s}^{-1}$.
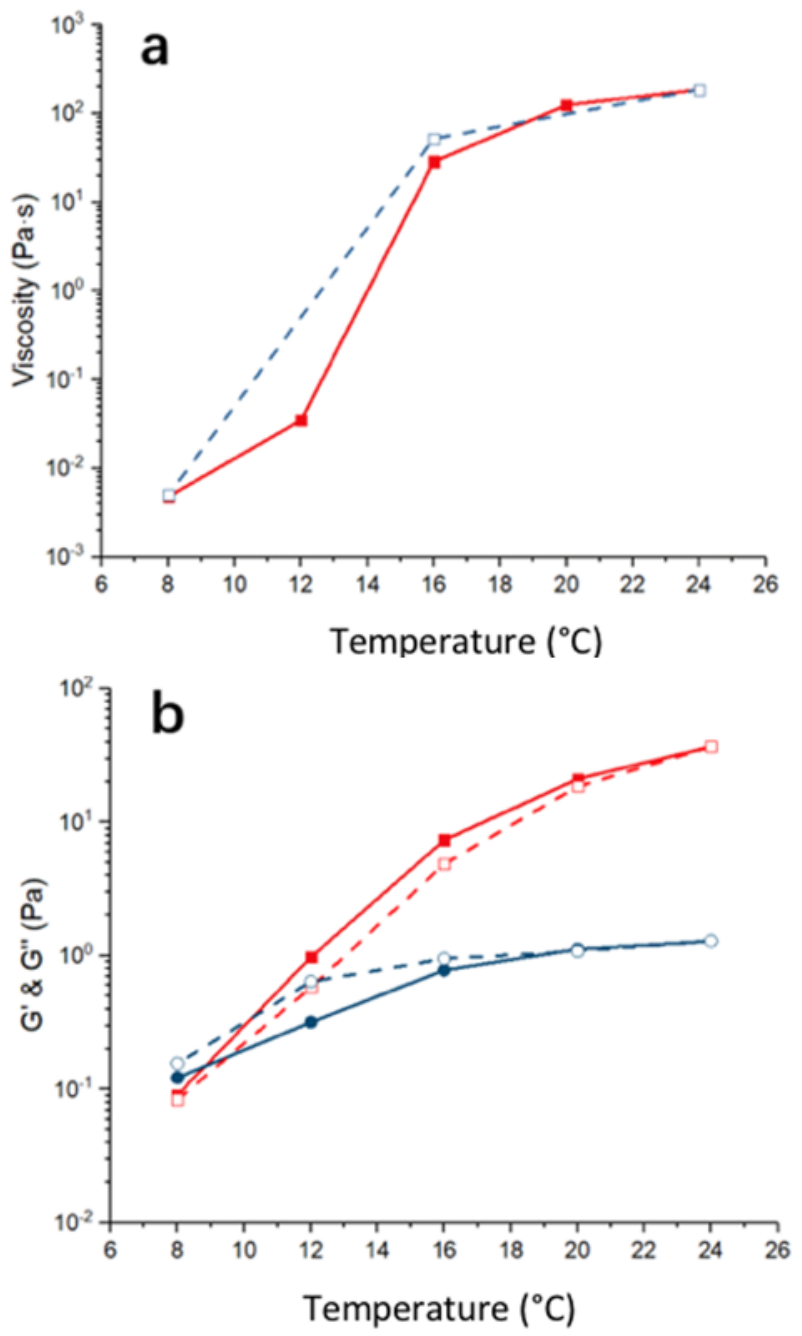

Figure 8. a) Viscosity of a 5 wt \% T5000-e-CNC-II sample during a $8-24{ }^{\circ} \mathrm{C}$ temperature cycle (heating: red squares and continuous line, and cooling: blue dots and dashed line) at a shear rate of $30 \mathrm{~s}^{-1}$; b) Storage modulus $G^{\prime}$ (red squares) and loss modulus $G^{\prime \prime}$ (blue dots) of a $5 \mathrm{wt} \%$ T5000-e-CNC-II sample during a $8-24{ }^{\circ} \mathrm{C}$ temperature cycle (heating: red squares and continuous line, and cooling: blue dots and dashed line) at 0.01 strain and an angular frequency of $1 \mathrm{~Hz}$.

cooled down even though the time interval between two measurements was tripled. When the temperature was decreased back to $8^{\circ} \mathrm{C}$, the initial viscosity value was recovered, showing a perfect thermoreversibility and a complete dissociation of the network upon cooling. These data confirm the reversibility observed by DLS and TEM. The evolution of the viscosity for the other four samples exhibits the same tendency but with different values (Supplementary Information Figures S8a-S11a). The higher the concentration, the higher the viscosity increase with temperature.

Figure $8 \mathrm{~b}$ shows the evolution of the storage, $G^{\prime}$, and loss, $G^{\prime \prime}$, moduli of a 5 wt \% T5000-e-CNC-II suspension as a function of temperature between 8 and $24^{\circ} \mathrm{C}$. Measurements were carried out at 0.01 strain and an angular frequency of $1 \mathrm{~Hz}$ after defining the strain and frequency corresponding to the linear domains. 
In the same way as for the viscosity, the viscoelastic behavior of non-modified CNC-II suspensions is insensitive to the temperature in the $8-24{ }^{\circ} \mathrm{C}$ range (Supplementary Information Figure S7b and S7c), in contrast with results reported by Yue et al. who observed an increase of $G^{\prime}$ when the temperature was increased from 15 to 25 and then $35^{\circ} \mathrm{C}$. ${ }^{13}$

For the T5000-e-CNC-II suspension, at $8^{\circ} \mathrm{C}, G^{\prime}$ is lower than $G^{\prime \prime}$ indicating a liquid-like (sol) behavior. As aforementioned, at such a temperature, entropic repulsive interactions prevent the formation of aggregates or connections and the nanoparticles were individual in the suspension. When the temperature increased toward the LCST of the polyetheramine, $G^{\prime}$ increased strongly and $G^{\prime \prime}$ increased more slowly. For $T>12{ }^{\circ} \mathrm{C}, G^{\prime}$ continued to increase, while $G$ " almost reached a plateau value at $1 \mathrm{~Pa}$ from $16^{\circ} \mathrm{C}$. $G^{\prime}$ is higher than $G^{\prime \prime}$ from $\mathrm{T}=12^{\circ} \mathrm{C}$ and the $G^{\prime} / G^{\prime \prime}$ ratio increases with the temperature to reach a value of about 30 at $24^{\circ} \mathrm{C}$. A temperature-induced transition from a liquid-like behavior to a gel-like behavior is therefore observed when the temperature is increased above the LCST of the polyetheramine. The gel behavior is attributed to attractive hydrophobic interactions between collapsed polymer chains at the extremities of the rods, which result in end-to-end connections of the rods and network formation. Interestingly, when the sample was cooled down from 24 to $8^{\circ} \mathrm{C}, \mathrm{G}^{\prime}$ and $G^{\prime \prime}$ decreased and recovered almost their value before heating. A full reversibility is thus observed and the cooling induces a transition from a gel to a sol following the breakage of end-toend associations and the recovery of individual repulsive particles. This result is in full agreement with DLS, TEM and viscosity data. The same tendency was found for the other four concentrations, but the higher the concentration, the stronger the effects (Supplementary Information Figures S8b-S11b).

This viscoelastic behavior observed here is different from that of suspensions of polyetheramine-grafted CNC-I particles, where the thermosensitive chains were decorating the whole surface of the rods, as reported by Azzam et al. ${ }^{41}$ In their case, CNC-I particles were grafted with the thermosensitive M2005 polymer.

When the temperature was above the LCST, these nanocrystals aggregated into bundles through attractive interaction forces between hydrophobic polymer chains on the lateral surfaces of the nanocrystals and the subsequent connection of the bundles resulted in a gelation. The variation of $G^{\prime}$ and $G^{\prime \prime}$ during the increase of temperature from below to above the LCST had a similar tendency to ours. However, the $G^{\prime} / G^{\prime \prime}$ ratio $(\tan \delta)$ in that case was limited to 3 , while it reached 30 in the case of T5000e-CNC-II. In fact, the gelation caused by CNCs aggregates in the case of the entire nanocrystal surface modification is due to the hydrophobic interactions between collapsed polymer chains adding interaggregate connection at a high concentration of nanoparticles. In the present work, a greater number of connections can be achieved as the CNC-II can associate through their ends to form a 3-dimensional network extending over tens of micrometers. The thermoreversible character of the gelation could be observed in both cases. This comparison shows that the fine tuning of the grafting loci allows controlling the rheological properties and the mechanisms of the gelation phenomenon.

\section{Conclusions}

In this work devoted to the regioselective functionalization of cellulose II nanocrystals, we first transposed the labeling with gold nanoparticles through an in situ growth strategy from the case of CNC-I to that of CNC-II and obtained a close to full conversion from rods to dumbbells particles comprising CNC-II functionalized with one AuNP at each end. This result provides a visual and statistically relevant proof of the antiparallel arrangement of cellulose chains in CNC-II and confirms the possible regioselective modification of the CNC-II ends only. It further provides an efficient method to yield hybrid particles that could benefit from synergistic properties. We secondly took advantage of the presence of reactive groups located at both ends of CNC-II to symmetrically functionalize these biosourced rods with thermosensitive polymer chains on both rod extremities. To achieve such a derivatization, a simple twostep chemical pathway was followed. Thanks to this derivatization, the colloidal behavior of CNC-II was modified and became temperature-sensitive. Below the LCST of polyetheramine chains, repulsive entropic interactions between swollen chains located at the rod ends add to the electrostatic repulsion provided by the surface sulfate half-esters on the lateral sides of the nanocrystals, yielding purely repulsive particles. In these conditions, the derivatized particles are individual and the resulting suspensions exhibit a shear-thinning behavior. When the temperature is increased above the LCST of the polymer, attractive interactions between collapsed hydrophobic chains develop, allowing end-to-end associations to take place and leading to the spectacular formation of a network extending over tens of micrometers, as observed from TEM images. From a macroscopic point of view, rheological measurements evidence the temperature-induced sol-gel transition from a liquid-like (sol) to a gel-like behavior, which is characterized by a high $G^{\prime} / G^{\prime \prime}$ ratio. All the experimental techniques used, namely DLS, TEM, viscosity and viscoelasticity measurements, consistently show a full reversibility upon sample cooling due to breakage of end-to-end associations when polymer-polymer interactions are turned to repulsive. Overall, the possibility to chemically modify both ends of CNC-II provides new properties when compared to the reducing-end modification of native $\mathrm{CNCs}$, further revealing the interest of the cellulose II allomorph for alternative and more extended functionalities that could not be reached with CNC-I. 


\section{Experimental section}

Cotton linters were provided by Buckeye Cellulose Corporation and used as the cellulose source without any further purification. Jeffamine ${ }^{\circledR}$ polyetheramine $T 5000$ (gift from the Huntsman Corporation), with a LCST of $\sim 12{ }^{\circ} \mathrm{C}$, is a tri-branched polymer of propylene glycol with amine end groups at each of its branches (chemical structure and evaluation of the LCST shown in Supplementary Information Figure S12) and a molecular weight of about $5000 \mathrm{~g} \mathrm{~mol}^{-1}$. Acetic acid (HAc) was purchased from Carlo Erba Reagents and other chemicals were purchased from Sigma-Aldrich and used as received. Deionized water was used in all experiments.

\section{Preparation of CNC-II by mercerization and acid hydrolysis}

CNC-II suspensions were prepared according to the method described by Flauzino Neto et al. ${ }^{12}$ Cotton linters were treated with a 20 wt \% sodium hydroxide aqueous solution under mechanical stirring for $5 \mathrm{~h}$ at $25^{\circ} \mathrm{C}$, using $30 \mathrm{~mL}$ of solution per gram of material. The material was washed several times with distilled water until the alkali was completely removed, and dried at $40^{\circ} \mathrm{C}$ for $12 \mathrm{~h}$ in an aircirculating oven. The mercerized cotton linter was milled and submitted to sulfuric acid hydrolysis. Here, for each gram of mercerized cotton linter, $20 \mathrm{~mL}$ of a $60 \mathrm{wt} \% \mathrm{H}_{2} \mathrm{SO}_{4}$ were used. The hydrolysis was performed at $45^{\circ} \mathrm{C}$ for 50 min under vigorous stirring. The suspensions were washed by repeated centrifugations, dialyzed against distilled water until constant conductivity of the dialysis bath. The suspensions were then ultrasonicated five times 2 min with a Branson 250 digital sonifier. The resulting suspension had a concentration of $2.4 \mathrm{wt} \%$.

Suspensions of higher concentration for rheological experiments were prepared as follows. The $\mathrm{pH}$ of a $1 \mathrm{wt} \% \mathrm{CNC}$-II suspension containing $3 \mathrm{~g}$ of dry CNCs was adjusted to 7 by adding an $0.1 \mathrm{M}$ $\mathrm{NaOH}$ solution. The suspension was then freeze-dried and the CNC-II were redispersed in distilled water to prepare aqueous CNC-II samples at a given concentration in the 1-5 wt \% range. Sample homogeneity was ensured by ultrasonicating three times for $2 \mathrm{~min}$.

\section{Sulfur content}

A suspension of CNC containing about $100 \mathrm{mg}$ of cellulose was titrated with a solution of $0.01 \mathrm{M} \mathrm{NaOH}$ using a MeterLab ${ }^{\circledR}$ CDM 210 conductivity meter. At the equivalence we have:

$$
V_{e q} \times C_{N a O H}=n_{\text {cellulose }} \times d_{S} \text { (Equation 1) }
$$

with $V_{e q}$ the added volume of $\mathrm{NaOH}, n_{\text {cellulose }}$ the number of moles of glucose residues and $d_{S}$ the degree of substitution representing the number of sulfate groups per unit of glucose. And we know that:

$$
n_{\text {cellulose }}=\frac{m_{\text {cellulose }}}{M_{\text {ave }}} \text { (Equation 2) }
$$

where $m_{\text {cellulose }}$ is the dry weight of introduced CNC and $M_{\text {ave }}$ is the average molar mass of a substituted glucose residue which is given by the following relation:

$$
M_{\text {ave }}=162 \times\left(1-d_{S}\right)+242 d_{S}(\text { Equation 3) }
$$

where $162 \mathrm{~g} / \mathrm{mol}$ et $242 \mathrm{~g} / \mathrm{mol}$ are respectively the molar masses of a unit of anhydroglucose and a mono-sulfated unit. Equations 1 and 2 allow us to write:

$$
d_{S}=\frac{V_{e q} \times C_{N a O H} \times M_{\text {ave }}}{m_{\text {cellulose }}} \text { (Equation 4) }
$$

After solving the equations 3 and $4, M_{\text {ave }}$ was calculated from the following relation:

$$
M_{\text {ave }}=\frac{162}{1-80 \times \frac{V_{e q} \times \mathrm{C}_{\mathrm{NaOH}}}{m_{\text {cellulose }}}}(\text { Equation 5) }
$$

The sulfur content, $\tau_{S}$, was then calculated using the following relation:

$$
\tau_{S}=\frac{32 \times d_{S}}{M_{\text {ave }}}(\text { Equation 6) }
$$

\section{Aldehyde content}

The Fehling test was used to measure the aldehyde content. ${ }^{42}$ Two solutions $A$ and $B$ were prepared. For solution $A, 69.28 \mathrm{~g}$ of $\mathrm{CuSO}_{4} \cdot 5 \mathrm{H}_{2} \mathrm{O}$ were dissolved in $1 \mathrm{~L}$ of distilled water. Solution $\mathrm{B}$ were prepared by dissolving $346 \mathrm{~g}$ of $\mathrm{KNaC}_{4} \mathrm{H}_{4} \mathrm{O}_{6} \bullet 4 \mathrm{H}_{2} \mathrm{O}$ and $120 \mathrm{~g}$ of $\mathrm{NaOH}$ in $1 \mathrm{~L}$ of distilled water. The final solution for the titration was obtained by mixing these two solutions in equal volume. $2 \mathrm{~g}$ of freeze dried CNCs were dispersed into $50 \mathrm{~mL}$ of deionized water. The suspension was heated to $65^{\circ} \mathrm{C}$ with a very gentle stirring and then titrated dropwise with the titration solution using a micropipette until there was no more new red precipitate. After each drop, an about 2 minutes delay was allowed for the detection of the red precipitate.

\section{Labeling of CNC-II with gold nanoparticles}

First, sulfur atoms were introduced through a reaction between the aldehydes at the reducing ends and thiosemicarbazide molecules. To do so, an aqueous solution containing $10 \mathrm{wt} \% \mathrm{HAc}$ and $2 \mathrm{wt} \%$ thiosemicarbazide was prepared. Then, a suspension containing about $2 \mathrm{~g}$ of CNC-II was mixed with the previous solution in the same volume in a flask surmounted by a condenser. The mixture was heated at $60^{\circ} \mathrm{C}$ for $3 \mathrm{~h}$ and finally cooled in ice. Then, $\mathrm{NaCl}$ was added until its concentration was approximately $0.4 \mathrm{M}$ and the suspension was centrifuged for $30 \mathrm{~min}$ at $11200 \mathrm{rpm}$ and the modified CNC-II were redispersed in HAc. The latter operation was repeated twice. The product was dialyzed against distilled water until constant conductivity of the dialysis bath and ultrasonicated for $4 \mathrm{~min}$ with a Branson 250 digital sonifier.

In a second step, an in situ growth method was performed to label the CNC-II ends with gold nanoparticles as follows. Two grams of a 1 wt \% sulfur-functionalized CNC-II suspension was added into $925 \mathrm{~mL}$ deionized water. The suspension was then heated to $95^{\circ} \mathrm{C}$. $92.5 \mathrm{mg}$ of chloroauric acid and $25 \mathrm{~mL}$ of a $1 \mathrm{wt} \%$ trisodium citrate aqueous solution were then added under stirring for $30 \mathrm{~min}$ at $95{ }^{\circ} \mathrm{C}$. The resulting suspension was then cooled down to room temperature. For comparison purposes, a suspension of AuNP was prepared following the procedure in the previous paragraph but in the absence of derivatized CNCs. 
Carboxylation of the reducing end of cellulose nanocrystals by chlorite oxidation

$\mathrm{NaClO}_{2}(5.652 \mathrm{~g}, 0.05 \mathrm{~mol})$ was added to a $2.4 \mathrm{wt} \%$ aqueous CNC suspension containing $1 \mathrm{~g}$ dry $\mathrm{CNC}$. HAc was then added until the $\mathrm{pH}$ reached 3.5 and the suspension was kept for $48 \mathrm{~h}$ under magnetic stirring. The reaction was stopped by adding a $0.5 \mathrm{M} \mathrm{NaOH}$ solution to reach a $\mathrm{pH}$ of about 8 . The suspension was dialyzed against distilled water until constant conductivity of the dialysis bath.

\section{Polymer grafting by peptide coupling}

Jeffamine ${ }^{\circledR}$ Polyetheramine T5000 (0.44 g, $\left.0.088 \mathrm{mmol}\right)$ was added to an 1 wt \% end-carboxylated CNC-II suspension, containing $0.5 \mathrm{~g}$ dry material and stirred until dissolution. The reaction was performed at $4{ }^{\circ} \mathrm{C}$ (well below the LCST) to ensure a good solubility of the polymer. The catalysts dicyclohexylcarbodiimide (DCC, 27.3 $\mathrm{mg}, 0.132 \mathrm{mmol}$ ) and 4-dimethylaminopyridine (DMAP, $10.75 \mathrm{mg}$, $0.088 \mathrm{mmol}$ ) were then added. After $24 \mathrm{~h}$ under stirring, the resulting suspensions, referred to as T5000-e-CNC-II, were filtered through $100 \mu \mathrm{m}$ nylon filters to get rid of the catalyst and dialyzed against distilled water to remove excess reagents including non-grafted polymers.

\section{Dynamic light scattering (DLS)}

DLS experiments were carried out with a Malvern NanozS instrument. All measurements were made at a well-controlled $( \pm$ $0.05{ }^{\circ} \mathrm{C}$ ) temperature with a $632.8 \mathrm{~nm}$ laser at a backscattering detection angle of $173^{\circ}$. The intensity size distribution was obtained from the analysis of the correlation function using the multiple narrow mode algorithm of the Malvern DTS software. For temperature effects investigation, heating and cooling rates were equal to 4 and $6^{\circ} \mathrm{C} \mathrm{min}^{-1}$, respectively.

\section{Transmission electron microscopy (TEM)}

Drops of about $0.001 \mathrm{wt} \% \mathrm{CNC}$-II aqueous suspensions were deposited onto carbon-coated TEM grids freshly glow-discharged in an EasiGlow plasma cleaning system (Pelco). After 2 min, the liquid in excess was blotted away with filter paper and, prior to drying, a drop of a 2 wt \% uranyl acetate aqueous solution was deposited on the specimen. After $2 \mathrm{~min}$, the stain in excess was wicked off, and the remaining thin liquid film allowed to dry. This protocol was adapted to account for the behavior of the grafted Jeffamine (LCST $\sim 12{ }^{\circ} \mathrm{C}$ ). One group of TEM grids were prepared in an ice-cooled vessel with a nanocrystal suspension and uranyl acetate solution both maintained at $\sim 4{ }^{\circ} \mathrm{C}$. Other grids were prepared from a nanocrystal suspension and uranyl acetate solution both preheated at $24^{\circ} \mathrm{C}$. The specimens were observed with a JEOL JEM 2100 Plus microscope operated at 200 kV. The images were recorded with a RIO 16 Gatan $(4096 \times 4096$ pixels) camera.

\section{Zeta potential}

The zeta potential of CNC-II was measured from $0.1 \mathrm{wt} \%$ suspensions by electrophoresis coupled with laser Doppler velocimetry using a Malvern NanozS instrument. Samples were measured in $10 \mathrm{mM}$ $\mathrm{NaCl}$. Data were averaged over 3 measurements, each measurement comprising 10 subruns.

\section{Small-Angle Neutron Scattering (SANS)}

SANS experiments were performed at Laboratoire Léon Brillouin (CEA-CNRS, Saclay, France) on the PAXY instrument. Four wavelength/sample-detector distance configurations ( $4 \AA$ at $1 \mathrm{~m}, 6 \AA$ at $3 \mathrm{~m}, 8.5 \AA$ at $5 \mathrm{~m}$ and $15 \AA$ at $6.7 \mathrm{~m}$ ) were used to access a momentum transfer range $\Delta Q$ from $2.10^{-3} \AA^{-1}$ to $0.6 \AA^{-1}$. The radially averaged spectra were corrected for solvent, empty cell, incoherent scattering and background noise to yield absolute intensity I vs. scattering vector $Q$ plots. To extract the dimensions of the particles, the curve corresponding to the prepared CNC-II suspension was fitted using the form factor of a parallelepiped with a rectangular section, averaged over all space orientations. The procedure used was the same as the one described by Cherhal et al. ${ }^{39}$ The polydispersity in this model was defined as the ratio of the half-width at half-maximum of the Gaussian distribution by the mean value of the dimension considered.

\section{Viscosity measurements}

The rheological behavior of aqueous CNC-II and polymer grafted CNC-II suspensions were carried out in steady state with a DHR3 rheometer from TA Instruments using a cone and plate geometry (angle 0 ${ }^{\circ} 59^{\prime} 42^{\prime \prime}$, diameter $60.0 \mathrm{~mm}$, gap $29 \mu \mathrm{m}$ ). Viscosity measurements were performed in a shear rate range from 0.01 to $1000 \mathrm{~s}^{-1}$. Steady flow were obtained by setting a shear rate until viscosity balance was reached. The ionic strength was controlled by adding $\mathrm{NaCl}$ solution until a $1 \mathrm{mM}$ concentration was reached. The evolution of viscosity as a function of temperature was investigated by varying the temperature from 8 to $24^{\circ} \mathrm{C}\left(1{ }^{\circ} \mathrm{C} \mathrm{min}-1\right.$ temperature ramp) on a Peltier plate at a shear rate of $30 \mathrm{~s}^{-1}$. Distilled water was used to saturate the atmosphere around the sample in order to avoid evaporation during the temperature ramp.

\section{Viscoelasticity measurements}

The DHR3 rheometer from TA Instruments was used to measure the viscoelastic properties of CNC-II and polymer grafted CNC-II suspensions. The ionic strength was controlled by adding $\mathrm{NaCl}$ solution until a $1 \mathrm{mM}$ concentration was reached. Experiments were carried out in the oscillatory mode with a cone and plate geometry (angle 0 $59^{\prime} 42^{\prime \prime}$, diameter $60.0 \mathrm{~mm}$, gap $29 \mu \mathrm{m}$ ). In order to determine the evolutions of the storage and loss moduli $G^{\prime}$ and $G^{\prime \prime}$ in a temperature range from 8 to $24{ }^{\circ} \mathrm{C}$, a Peltier plate that allowed varying the temperature was used and the same setup as for the viscosity measurements was used to avoid evaporation. The temperature ramp was $3{ }^{\circ} \mathrm{C} \mathrm{min}-1$ and delay times of $10 \mathrm{~min}$ or $30 \mathrm{~min}$ were applied after reaching the set point upon temperature increase or decrease, respectively. 
Strain sweep procedures were applied in the range of 0.001 to 3 at an angular frequency of $1 \mathrm{~Hz}$ to define the strain range corresponding to the linear domains of deformation. Then, angular frequency sweep procedures of $0.1-10 \mathrm{~Hz}$ were performed at a strain of 0.1 or 0.2 according to the samples to define the frequency value. For the tests of temperature effect, the sweep procedures were carried out at 8 and $24{ }^{\circ} \mathrm{C}$ to determine average values of strain and frequency corresponding to the linear domains for each sample. These values were used along the temperature range. The manuscript was written through contributions of all authors. All authors have given approval to the final version of the manuscript.

\section{Author contributions}

The manuscript was written through contributions of all authors. All authors have given approval to the final version of the manuscript.

\section{Conflicts of interest}

There are no conflicts to declare.

\section{Acknowledgements}

The authors acknowledge the NanoBio-ICMG chemistry platform (FR 2607, Grenoble) for granting access to the electron microscopy and NMR facilities, and Laboratoire Léon Brillouin (LLB, CEA Saclay, France) for neutron beamtime allocation. B.J. thanks Fabrice Cousin for impeccable neutron service at LLB throughout the years. CERMAV is part of LabEx Arcane and CBHEUR-GS (Investissements d'Avenir - grant agreement \#ANR-17EURE-0003). LRP is part of LabEx Tec21 (Investissements d'Avenir - grant agreement no. ANR-11-LABX-0030) and. Both laboratories are part of PolyNat Carnot Institut (Investissements d'Avenir - grant agreement \#ANR-16-CARN-0025-01). Part of this work was developed within the framework of Glyco@Alps, supported by Agence Nationale de la Recherche (Investissements d'Avenir, grant agreement \#ANR-15-IDEX-02).

\section{Notes and references}

1. D. Klemm, B. Heublein, H. P. Fink and A. Bohn, Angew. Chem. Int. Edit., 2005, 44, 3358-3393.

2. A. Dufresne, Nanocellulose: from nature to high performance tailored materials, Walter de Gruyter GmbH, Berlin, 2012.

3. K. H. Gardner and J. Blackwell, Biopolymers, 1974, 13, 19752001.

4. I. A. Sacui, R. C. Nieuwendaal, D. J. Burnett, S. J. Stranick, M. Jorfi, C. Weder, E. J. Foster, R. T. Olsson and J. W. Gilman, ACS Appl. Mater. Interfaces, 2014, 6, 6127-6138.
5. H. Chanzy and B. Henrissat, FEBS Lett., 1985, 184, 285-288.

6. K. Hieta, S. Kuga and M. Usuda, Biopolymers, 1984, 23, 18071810.

7. T. Imai, T. Watanabe, T. Yui and J. Sugiyama, FEBS Lett., 2002, 510, 201-205.

8. S. Kuga and R. M. Brown, Carbohydr. Res., 1988, 180, 345-350. 9. J. F. Revol, H. Bradford, J. Giasson, R. H. Marchessault and D. G. Gray, Int. J. Biol. Macromol., 1992, 14, 170-172.

10. Br. Pat., Patent 13296, 1850.

11. N. H. Kim, T. Imai, M. Wada and J. Sugiyama, Biomacromolecules, 2006, 7, 274-280.

12. W. P. Flauzino Neto, J. L. Putaux, M. Mariano, Y. Ogawa, H. Otaguro, D. Pasquini and A. Dufresne, RSC Adv., 2016, 6, 76017-76027.

13. Y. Y. Yue, C. J. Zhou, A. D. French, G. Xia, G. P. Han, Q. W. Wang and Q. L. Wu, Cellulose, 2012, 19, 1173-1187.

14. G. Delepierre, S. Eyley, W. Thielemans, C. Weder, E. D. Cranston and J. O. Zoppe, Nanoscale, 2020, 12, 17480-17493.

15. J. Gong, J. Li, J. Xu, Z. Y. Xian and L. H. Mo, RSC Adv., 2017, 7, 33486-33493.

16. M. Hirota, N. Tamura, T. Saito and A. Isogai, Cellulose, 2010, 17, 279-288.

17. M. Hirota, N. Tamura, T. Saito and A. Isogai, Cellulose, 2012, 19, $435-442$

18. J. Gong, L. H. Mo and J. Li, Carbohydr. Polym., 2018, 195, 1828.

19. G. Sèbe, F. Ham-Pichavant, E. Ibarboure, A. L. C. Koffi and P. Tingaut, Biomacromolecules, 2012, 13, 570-578.

20. E. Jin, J. Q. Guo, F. Yang, Y. Y. Zhu, J. L. Song, Y. C. Jin and O. J. Rojas, Carbohydr. Polym., 2016, 143, 327-335.

21. D. F. Martins, A. B. de Souza, M. A. Henrique, H. A. Silvério, W. P. F. Neto, D. J. I. C. Pasquini and Products, 2015, 65, 496-505.

22. M. A. Henrique, W. P. Flauzino Neto, H. A. Silvério, D. F. Martins, L. V. A. Gurgel, H. da Silva Barud, L. C. de Morais and D. Pasquini, Ind Crop Prod, 2015, 76, 128-140.

23. H.-Y. Yu, H. Zhang, M.-L. Song, Y. Zhou, J. Yao, , Q.-Q. Ni, ACS Appl. Mater. Interfaces, 2017, 9, 43920-43938.

24. X. Li, J. Li, J. Gong, Y. Kuang, L. Mo and T. Song, Carbohydrate Polymers, 2018, 183, 303-310.

25. Y. S. Kuang, X. Li, P. C. Luan, X. Zhang, J. Xu, L. H. Mo, J. Gong and J. Li, Cellulose, 2020, 27, 3167-3179.

26. F. Lin, F. Cousin, J. L. Putaux and B. Jean, ACS Macro Lett., 2019, 8, 345-351.

27. K. Heise, G. Delepierre, A. W. T. King, M. A. Kostiainen, J. O. Zoppe, C. Weder and E. Kontturi, Angew. Chem.-Int. Edit., 2020, DOI: 10.1002/anie.202002433.

28. H. Tao, N. Lavoine, F. Jiang, J. T. Tang and N. Lin, Nanoscale Horiz., 2020, 5, 607-627

29. A. D. French, Cellulose, 2014, 21, 885-896.

30. S. Y. Oh, D. I. Yoo, Y. Shin, H. C. Kim, H. Y. Kim, Y. S. Chung, W. H. Park and J. H. Youk, Carbohydr. Res., 2005, 340, 2376-2391. 
31. P. Dhar, D. Tarafder, A. Kumar and V. J. R. a. Katiyar, 2015, 5, 60426-60440.

32. J. Q. Han, C. J. Zhou, A. D. French, G. P. Han and Q. L. Wu, Carbohydr. Polym., 2013, 94, 773-781.

33. R. Zuluaga, J. L. Putaux, J. Cruz, J. Vélez, I. Mondragon and P. J. C. P. Gañán, 2009, 76, 51-59.

34. S. Park, D. K. Johnson, C. I. Ishizawa, P. A. Parilla and M. F. Davis, Cellulose, 2009, 16, 641-647.

35. F. Azzam, B. Frka-Petesic, E. F. Semeraro, F. Cousin and B. Jean, Langmuir, 2020, 36, 8511-8519.

36. S. Elazzouzi-Hafraoui, Y. Nishiyama, J.-L. Putaux, L. Heux, F. Dubreuil and C. Rochas, Biomacromolecules, 2008, 9, 57-65.

37. P. Terech, L. Chazeau and J. Y. Cavaille, Macromolecules, 1999, 32, 1872-1875.

38. F. Cherhal, F. Cousin and I. Capron, Langmuir, 2015, 31, 55965602.

39. Y. M. Mao, K. Liu, C. B. Zhan, L. H. Geng, B. Chu and B. S. Hsiao, J. Phys. Chem. B, 2017, 121, 1340-1351.

40. F. Azzam, E. Siqueira, S. Fort, R. Hassaini, F. Pignon, C. Travelet, J. L. Putaux and B. Jean, Biomacromolecules, 2016, 17, 21122119.

41. K. P. C. Vollhardt and N. E. Schore, Organic Chemistry, Macmillan International Higher Education, 2014. 